\title{
Effect of Stimulus Polarity on Detection Thresholds in Cochlear Implant Users: Relationships with Average Threshold, Gap Detection, and Rate Discrimination
}

\author{
Robert P. Carlyon, ${ }^{1}$ (D Stefano Cosentino, ${ }^{1}$ John M. Deeks, ${ }^{1}$ Wendy Parkinson, ${ }^{2}$ and \\ Julie A. Arenberg ${ }^{2}$ \\ ${ }^{1}$ Medical Research Council Cognition and Brain Sciences Unit, University of Cambridge, 15 Chaucer Road, Cambridge, CB2 \\ $7 E F, U K$ \\ ${ }^{2}$ Department of Speech and Hearing Sciences, University of Washington, 1417 NE 42nd St., Seattle, WA 98105, USA
}

Received: 1 November 2017; Accepted: 18 May 2018; Online publication: 7 June 2018

\begin{abstract}
Previous psychophysical and modeling studies suggest that cathodic stimulation by a cochlear implant (CI) may preferentially activate the peripheral processes of the auditory nerve, whereas anodic stimulation may preferentially activate the central axons. Because neural degeneration typically starts with loss of the peripheral processes, lower thresholds for cathodic than for anodic stimulation may indicate good local neural survival. We measured thresholds for 99-pulse-per-second trains of triphasic (TP) pulses where the central high-amplitude phase was either anodic (TP-A) or cathodic (TP-C). Thresholds were obtained in monopolar mode from four or five electrodes and a total of eight ears from subjects implanted with the Advanced Bionics CI. When between-subject differences were removed, there was a modest but significant correlation between the polarity effect (TP-C threshold minus TP-A threshold) and the average of TP-C and TP-A thresholds, consistent with the hypothesis that a large polarity effect corresponds to good neural survival. When data were averaged across electrodes for each subject, relatively low thresholds for TP-C correlated with a high "upper limit" (the pulse rate up to which pitch continues to increase) from a previous study (Cosentino et al. J Assoc Otolaryngol 17:371-382). Overall, the results provide modest indi-
\end{abstract}

Correspondence to: Robert P. Carlyon - Medical Research Council Cognition and Brain Sciences Unit - University of Cambridge 15 Chaucer Road, Cambridge, CB2 7EF, UK. Telephone: +44 1223 355294; email: bob.carlyon@mrc-cbu.cam.ac.uk rect support for the hypothesis that the polarity effect provides an estimate of local neural survival.

Keywords: cochlear implants, polarity effects, detection thresholds

\section{INTRODUCTION}

Many cochlear implant (CI) listeners understand speech well, at least in quiet. However, there remains substantial across-listener variability, with some struggling even in favorable listening conditions (Holden et al. 2013). In recent years, there has been considerable interest in identifying the reasons for poor speech perception, and in identifying the relationship between performance and the global and local pattern of neural survival in each individual patient. A potentially useful approach comes from singleelectrode psychophysical measures, which have revealed substantial across-listener and across-electrode variability in a number of tasks. These include signal detection in quiet and supra-threshold measures. Authors have investigated the variation in not only the absolute level of thresholds (Pfingst and Xu 2004; Bierer et al. 2015) but also how they are influenced by pulse rate ("multi-pulse integration, MPI"; Zhou and Pfingst 2014; Zhou et al. 2015; Zhou and Pfingst 2016), pulse polarity (Macherey et al. 2017), and stimulation mode (Bierer 2007; Bierer and Faulkner 2010). Supra-threshold tasks have included modula- 
tion detection (Garadat et al. 2012; Garadat et al. 2013), gap detection (Bierer et al. 2015), and rate discrimination both at low and high rates (Cosentino et al. 2016). It is worth noting that variation in all of these measures has been observed even when stimulating in monopolar mode, which is believed to produce a wide spread of excitation within the cochlea. Indeed, even though the variation in thresholds across the electrode array is smaller in $\mathrm{dB}$ for monopolar than for tripolar stimulation, this is not true when the across-electrode standard deviation (s.d.) is normalized by the within-electrode s.d. (Bierer et al. 2015). In other words, monopolar stimulation may reveal across-electrode variation as reliably as tripolar stimulation, even though the size of this variation is smaller in monopolar than in tripolar mode when expressed in $\mathrm{dB}$.

A potentially important application of singleelectrode measures is that they may guide the clinician in choosing which, if any, electrodes to deactivate when optimizing patient maps. Indeed, significant improvements in speech perception scores have been obtained by deactivating electrodes based on highmodulation detection thresholds (Garadat et al. 2012; Garadat et al. 2013) and high thresholds for low-rate pulse trains (Zhou 2017). In order to provide a principled approach to channel selection, it would be useful to know how the various different single-electrode measures correlate with each other. This could then either reveal clusters of tests, each of which taps a particular consequence of neural degeneration, or reveal a single test factor that could be used to guide channel selection algorithms. This information may also provide basic insights into the limitations of hearing by CI users. For example, Cosentino et al. (2016) found that the "upper limit" of temporal pitch-defined as the highest pulse rate on a single electrode above which pitch no longer increased-correlated significantly with gap detection thresholds (GDTs), but not with the smallest difference in the rate of a low-rate pulse train that could be discriminated. The significant difference between these two correlations led them to suggest that there is a limitation specific to tasks that require sustained temporally accurate firing to high pulse rates, and which is separate from that which limits low-rate discrimination. Zhou and Pfingst (2016) reported that MPI correlated significantly with the degree of spatial selectivity for a given electrode, and concluded that integration of multiple pulses is most efficient when conveyed by neurons that innervate a wide region of the cochlea.

The present study forms part of a series that compares performance on different single-electrode psychophysical measures in a group of CI users (Bierer et al. 2015; Cosentino et al. 2016). Here, we measure polarity sensitivity, defined as the difference between thresholds for 99-pps trains of triphasic pulses in which the short high-amplitude portion is either anodic or cathodic. As with other types of asymmetric pulse, triphasic stimulation allows one to study polarity sensitivity by concentrating charge of one polarity into a short time period, while maintaining the charge balancing necessary for patient safety. All stimulation is in monopolar mode. The motivation stems from the finding that, although animal studies usually reveal greater sensitivity to cathodic than to anodic stimulation (Hartmann et al. 1984; Miller et al. 1999; Miller et al. 2004), the reverse is true for human CI users when presented with stimuli at or close to their most comfortable listening level ("MCL"; Macherey et al. 2006; Macherey et al. 2008; van Wieringen et al. 2008; Undurraga et al. 2010; Macherey et al. 2011). A possible reason for this discrepancy, consistent with computational models (Rattay 1999; Rattay et al. 2001), is that cathodic stimulation depolarizes the peripheral processes of the auditory nerve. These processes are likely to be intact in the recently deafened animals used in most physiological experiments. However, there is evidence that peripheral processes are more susceptible than central axons to auditory deprivation, and so may have deteriorated in human CI users who have been deaf for months or years prior to implantation (Johnsson et al. 1981).

Stimulus polarity can affect not only MCLs but also thresholds. Unlike MCL measures, the direction of the polarity sensitivity at threshold varies consistently across listeners and electrodes, and some electrodelistener combinations reveal lower thresholds for cathodic than for anodic stimulation (Macherey et al. 2017; Mesnildrey et al. 2017). These combinations may reflect local regions of good neural survival in which a relatively high proportion of peripheral processes remain. Here, we investigate whether this putative measure of neural survival correlates with measures of gap detection, low-rate discrimination, and the upper limit of temporal pitch obtained in our previous studies (Bierer et al. 2015; Cosentino et al. 2016). The hypothesis is that lower thresholds for cathodic than for anodic stimulation will correlate with tasks that depend on good local neural survival. Note, however, that this does not require that the variation in performance on those tasks is limited by the pattern of activity in the auditory nerve; rather, poor auditory nerve survival may lead to more central degeneration which in turn could limit performance on perceptual tasks. For example, Carlyon and Deeks (2015) found that the "alternating-amplitude" pattern of auditory nerve-evoked responses (Wilson 1997) to high-rate pulse trains correlated across subjects with poor rate discrimination at high rates, 
but also demonstrated that the correlation was not causal, and that manipulating the stimulus so as to reduce the alternating-amplitude pattern did not improve performance.

A second prediction is that polarity sensitivity will correlate with the average of the thresholds in the two polarities, on the assumption that better sensitivity to cathodic stimulation will reflect better neural survival and hence lower overall thresholds. Specifically, we assume that the average thresholds will also depend on the distance of the electrodes from the modiolus (electrode-modiolus distance, "EMD") but that the effects of EMD and polarity are independent. In the "Discussion" section, we describe a recent study that provides evidence for this assumption.

When comparing performance on different tasks, two types of measure are possible. One of these is to correlate performance across subjects (e.g., Fu 2002; Won et al. 2011; Cosentino et al. 2016). This can harness the often substantial across-listener variability in performance, but is potentially susceptible to nonspecific effects such as attention span and cognitive ability. Such effects could lead to a correlation that does not reflect any common processing of the two tasks, except at very central levels. A more rigorous approach is to partial out between-subject effects, and to correlate the relative pattern of scores across electrodes (Bierer 2007; Cosentino et al. 2015; Zhou and Pfingst 2016). This approach is immune to between-listener cognitive differences. Acrosselectrode differences are also of more clinical relevance because, as mentioned above, they may guide channel selection methods that aim to optimize performance on a listener-by-listener basis. However, because this type of analysis excludes the substantial variation in neural survival that occurs across listeners, for example due to differences in pathology (e.g., Zimmermann et al. 1995; e.g., Nadol 1997), it risks "throwing the baby out with the bath water". We perform both types of analysis here. One advantage of studying polarity sensitivity is that, being a difference between two thresholds, it is unlikely to be affected by between-listener variation in cognition. Hence, it may exploit the benefits of measuring the substantial across-listener variation in neural survival without being strongly influenced by cognitive effects.

\section{METHODS}

\section{Subjects}

Eight ears from seven post-lingually deafened adults wearing the Advanced Bionics HiRes90K CI were studied; listener details are shown in Table 1. One subject, who was bilaterally implanted, was tested in each ear and is listed as S30L and S39R in the table.

\section{TABLE 1}

Details of the listeners who took part. IDs starting with the letter $\mathrm{S}$ refer to patients implanted and tested in Seattle, USA. Those beginning with the letter $\mathrm{C}$ were implanted and tested in Cambridge, UK. "Subjects" S3OL and S39R refer to the left and right ears of the same participant and are treated separately throughout this article

\begin{tabular}{lllll}
\hline ID & $\begin{array}{l}\text { Age } \\
\text { (years) }\end{array}$ & $\begin{array}{l}\text { Deafness onset } \\
\text { (age, years) }\end{array}$ & $\begin{array}{l}\text { Possible } \\
\text { etiology }\end{array}$ & $\begin{array}{l}\text { Duration of Cl } \\
\text { use (years) }\end{array}$ \\
\hline S22 & 74 & 55 & Hereditary & 7 \\
S30 L & 51 & 16 & Hereditary & 11 \\
S39R & 51 & 16 & Hereditary & 31 \\
C1 & 69 & 32 & Unknown & 5 \\
C3 & 71 & 50 & Otosclerosis & 4 \\
C4 & 68 & 37 & Otosclerosis & 6 \\
C5 & 55 & 31 & Unknown & 6 \\
C6 & 66 & 51 & Unknown & 3 \\
\hline
\end{tabular}

This subject's two ears were treated as completely separate, and therefore, for the purposes of analysis and for discussion in the remainder of this article, there were eight "subjects." They had all participated in the study by Cosentino et al. (2016) and the same subject codes are used here. Five of the subjects were implanted and tested in Cambridge, UK, whereas the other three were implanted and tested in Seattle, WA, USA. All procedures were approved by the respective Human Subjects Review Boards.

\section{Stimuli}

Triphasic pulses were used, in which the amplitude of the central phase was twice that of each of the first and third phases. They are named here in terms of the central high-amplitude phase, which was anodic for stimulus TP-A and cathodic for stimulus TP-C. (Note that here we use the abbreviation "TP" to refer to the triphasic pulse shape rather than to tripolar stimulation mode as in some other articles.) Phase durations were $43 \mu \mathrm{s}$, the pulse rate was 99 pulses per second, and the duration of each pulse train was $400 \mathrm{~ms}$. Each subject was tested on the same four or five electrodes as used for the rate discrimination and gap detection measures by Cosentino et al. (2016). All stimuli were presented in monopolar mode. Initially, MCLs were obtained for each combination of subject and electrode, so as to guide the starting level for the threshold measurements and to set a safety limit for those procedures. After each stimulus was presented, the subject indicated its loudness using the Advanced Bionics loudness rating scale for which a " 6 " is "Most Comfortable" and a "7" is "Loud but Comfortable." The level was increased gradually until the rating was 7 and then reduced until the listener reported a 6 again. All stimuli were presented and controlled using research hardware and software ("BEDCS") provided 
by the Advanced Bionics company. Programs were written using the MATLAB programming environment, which controlled low-level BEDCS routines. Stimuli were checked using a test implant and digital storage oscilloscope. The same software and type of hardware were used at both testing sites (Cambridge and Seattle).

Signal detection thresholds were measured using a three-down, one-up, two-interval forced choice adaptive procedure that converged on $79 \%$ correct. Step size was $1 \mathrm{~dB}$ for the first two turnpoints and $0.25 \mathrm{~dB}$ thereafter. The mean of the last four of six turnpoints was used to estimate threshold. Four repetitions were performed for each measurement and then averaged. Subjects were asked "Which interval contained the sound?" and responded by selecting a button on a computer screen. Correct-answer feedback was provided at the end of each trial.

\section{RESULTS}

Thresholds (Ts) for each subject are shown for the TP-A (red triangles) and TP-C (blue circles) stimuli in Fig. 1. It can be seen that the polarity effect $\left(\mathrm{T}_{\mathrm{TP}-\mathrm{C}^{-}}\right.$ $\mathrm{T}_{\mathrm{TP}-\mathrm{A}}$ ) varies across subjects and electrodes. Of the 33 subject-electrode combinations, 9 showed a lower threshold for the TP-C than for the TP-A stimulus and hence a negative polarity effect. Recall that, according to our hypothesis, low or negative polarity effects reflect good neural survival.

\section{Between-Electrode Correlations}

To assess between-electrode correlations, we performed univariate ANOVAs with one factor as the dependent variable, the other as a covariate, and with subject entered as a random effect. This revealed a significant positive correlation between the polarity effect and the average of the TP-A and TP-C thresholds $(r=0.49, F(1,24)=7.59, p=0.011)$. Our statistical approach is mathematically equivalent to subtracting each subject's mean score from every data point, so as to obtain a normalized score, and then correlating these normalized scores. The resulting correlation is shown in Fig. 2. It is in the direction predicted by the hypothesis that lower/negative polarity effects and lower overall thresholds both reflect better survival of the peripheral processes. Note that, although the difference between two scores will a priori be correlated with each score alone, no such a priori relationship holds between the difference and the average of two scores, under the null hypothesis that the variances of each score are equal (Oldham 1962; Tu and Gilthorpe 2007). This is true even when the two scores correlate with each other. For example, if EMD and neural survival combine additively to affect thresholds, then the acrosselectrode variation in EMD will produce a correlation between TP-A and TP-C thresholds; however, this additive effect will not cause the mean and difference to correlate with each other.

Although there was a significant across-electrode correlation between the polarity effect and the average thresholds, there was no significant correlation between either of them and any of the suprathreshold measures obtained in our previous studies. Those measures were the $\log$ of the gap detection thresholds (GDTs) measured by Bierer et al. (2015), and two rate discrimination measures ("RDR100" and "RDR400") obtained by Cosentino et al. (2016). One of those, RDR100, is the log of the ratio between two just-discriminable pulse rates, where the standard had a rate of 100 pps and the signal, whose rate was always above 100 pps, was adaptively varied to obtain
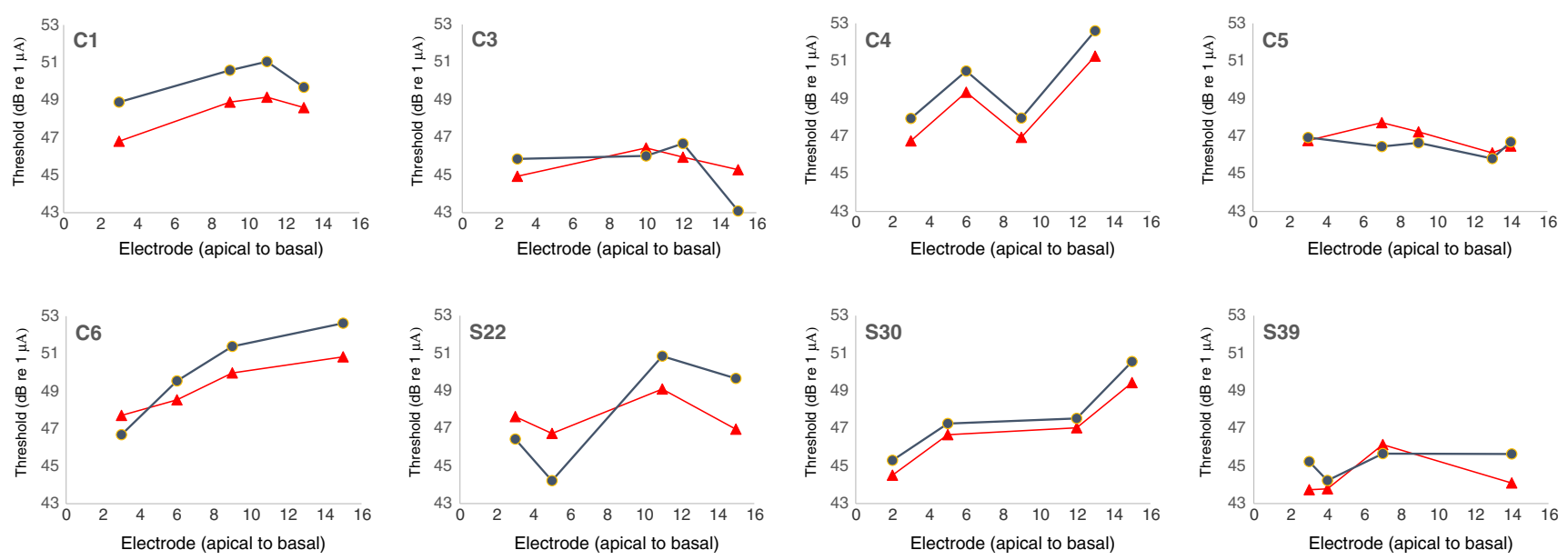

FIG. 1. Thresholds as a function of electrode number for each listener. Thresholds for TP-A stimuli are shown by red triangles, whereas those for TP-C stimuli are shown by blue circles 


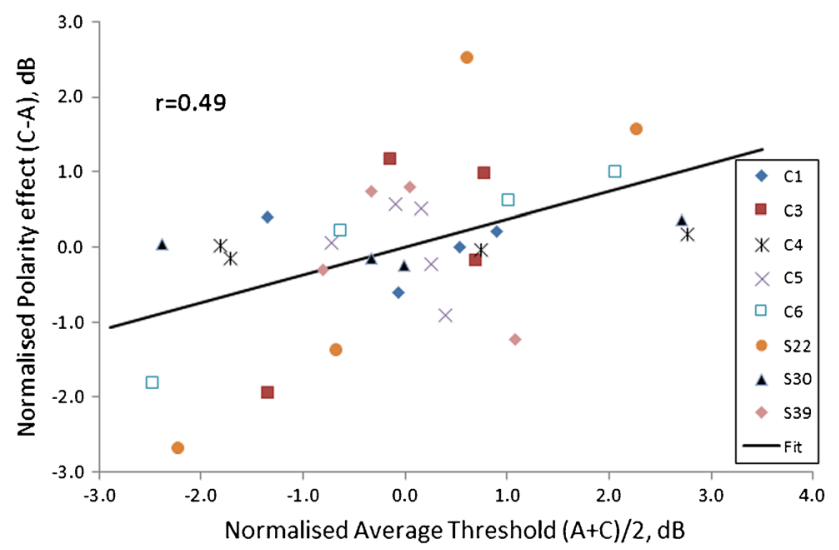

FIG. 2. Correlation between the average thresholds $(A+C / 2)$ and the difference between cathodic and anodic thresholds $(C-A)$, in decibels. Between-subject differences have been removed by subtracting the average value for each subject from every data point for that subject. This normalization removes the effects of betweensubject correlations, leaving only the between-electrode correlation. Data for each subject are shown by a unique symbol

threshold. The other, RDR400, was similar except that the standard had a rate of 400 pps and the signal always had a rate lower than 400 pps. The two measures reflect low-rate discrimination and the upper limit of temporal pitch, respectively. In both cases, low values represent better performance. The between-electrode correlations are shown in Table 2.

We also investigated the effect of the longitudinal electrode position in the array. Bierer (2007) reported a trend for thresholds to decrease from the base to the apex of the electrode array for symmetric biphasic pulses presented in partial tripolar mode, but not in bipolar or monopolar mode. They suggested that this could be due to the progression of spiral ganglion degeneration from base to apex in several pathologies (Zimmermann et al. 1995), and/or to a variation in the EMD along the array. Subsequently, Bierer et al.

\section{TABLE 2}

Across-electrode correlations between the average threshold across polarities and the polarity effect with the logarithms of gap detection thresholds (GDTs), and of rate discrimination measures at low (RDR100) and high (RDR400) rates. The correlations were obtained by subtracting each subject's mean score from every point, thereby removing betweensubject effects. This is equivalent to performing an analysis of covariance with one measure as the dependent variable, the other as a covariate, and subject as a random factor (Bland and Altman 1995). In all cases, there were 24 degrees of freedom. The $1 \%$ and $5 \%$ significance levels for $\mathrm{df}=24$ are, respectively, 0.50 and 0.39

\begin{tabular}{lrcc}
\hline & GDT & RDR100 & RDR400 \\
\hline Average & 0.24 & 0.09 & -0.35 \\
Polarity & -0.08 & 0.06 & -0.29 \\
\hline
\end{tabular}

(2015) found that thresholds decreased from base to apex for symmetric biphasic pulses presented both in partial tripolar and monopolar mode. A similar finding was observed in the present data for the average of the TP-A and TP-C thresholds, as assessed by a univariate ANOVA with average threshold as dependent variable, subject as a random effect, and electrode number as covariate $(F(1,24)=11.41, p=$ $0.002)$. However, no such finding was observed for the polarity effect $(F(1,24)=2.76, p=0.16)$. Hence, if the polarity effect reflects neural survival, the present results provide no evidence that, for this group of subjects, the effect of longitudinal electrode position on average thresholds was due to a variation in the pattern of neural survival.

\section{Between-Subject Correlations}

Between-subject correlations were obtained by calculating the average score across electrodes for each subject and then performing product-moment correlations. The correlation between the polarity effect and the average threshold was in the same direction as for the between-electrode correlations, but did not reach statistical significance $(r=0.60, \mathrm{df}=6, p=0.16)$. The correlations between each of these measures and the supra-threshold scores obtained previously (GDT, RDR100, and RDR400) are shown in Table 3. All of the correlations were positive, three reached statistical significance, and two did so after Bonferroni correction for multiple comparisons. One of these was between the average threshold and GDT $(r=0.87$, $\mathrm{df}=6, p=0.0050$, corrected $p=0.03)$. The other was between the polarity effect and RDR400 $(r=0.91$, df $=$ $6, p=0.0017$, corrected $p=0.01)$. As noted in the Introduction, it is unlikely that the second of these can be explained in terms of cognitive factors, such as the ability or willingness to concentrate on the task, because the polarity effect is a difference score.

\section{DISCUSSION}

\section{Comparison to Previous Studies}

A large number of psychophysical and electrophysiological studies have demonstrated CI users' greater sensitivity to anodic than to cathodic current for moderately and comfortably loud stimuli (Macherey et al. 2006; Macherey et al. 2008; van Wieringen et al. 2008; Undurraga et al. 2010; Macherey et al. 2011; Carlyon et al. 2013). More recently, two studies have shown that, although detection thresholds do not differ overall between stimuli of opposite polarity, individual CI listeners show idiosyncratic but consistent polarity effects. 


\section{TABLE 3}

Across-subject correlations between the average threshold across polarities and the polarity effect with the logarithms of gap detection thresholds (GDTs), and of rate discrimination measures at low (RDR100) and high (RDR400) rates. The correlation between the average threshold and the polarity effect was 0.6. Statistically significant correlations are shown in italics; those that survived Bonferroni corrections for six comparisons are additionally presented in boldface (see text for details). The number of degrees of freedom was 6 in all cases. The $1 \%$ and $5 \%$ significance levels for $\mathrm{df}=6$ are, respectively, 0.83 and 0.71

\begin{tabular}{lccc}
\hline & $G D T$ & $R D R 100$ & $R D R 400$ \\
\hline Average & $\mathbf{0 . 8 7}$ & 0.72 & 0.61 \\
Polarity & 0.41 & 0.49 & $\mathbf{0 . 9 1}$ \\
\hline
\end{tabular}

Macherey et al. (2017) measured thresholds for trains of symmetric biphasic pulses with either anodic or cathodic leading polarity ("SYM-A" and "SYM-C"), as well as for trains of so-called quadraphasic ("QP") pulses. The QP pulses were constructed by abutting two SYM pulses of opposite leading polarity, such that the central portion consisted of two anodic (QP-A) or cathodic (QP-C) phases. A previous study had shown that, as for the TP-A and TP-C pulse trains used here and for pseudomonophasic pulse trains, the loudness of supra-threshold QP-A pulse trains was consistently greater than that of QP-C pulse trains at the same current (Carlyon et al. 2013). Macherey et al. (2017) found that, across subjects, the difference between QP-A and QP-C thresholds correlated strongly with that between SYM-A and SYM-C thresholds, demonstrating that the idiosyncratic differences observed across subjects were not simply due to measurement noise. In addition, they reported that, for some electrode-listener combinations, the loudness growth function was non-monotonic, and that this unusual pattern was significantly more likely to occur when thresholds were lower for QP-C than for QP-A pulse trains. They suggested, as we do here, that low QP-C thresholds may reflect good local survival and activation of peripheral processes. They further suggested that non-monotonic loudness growth might then be explained by "cathodal block" (Ranck 1975) impeding the propagation of action potentials as level was increased. Interestingly, they only observed nonmonotonic growth for users of the Cochlear CI; when they tested five Advanced Bionics users on one electrode each, the loudness growth was always monotonic. The fact that we reported several instances of substantially negative polarity effects raises the possibility that, for those electrode-subject combinations, the loudness growth may also have been nonmonotonic. A stronger test of this hypothesis would involve measuring loudness growth functions for the subject-electrode combinations showing the most negative polarity effects, such as C3 (electrode 15, $2.2 \mathrm{~dB}$ ), C5 (electrode 7, $-1.3 \mathrm{~dB}$ ), and S22 (electrode $5,-2.45 \mathrm{~dB})$.

Mesnildrey et al. (2017) measured thresholds using a fast one-interval adjustment procedure for trains of TP-A and TP-C pulses presented in partial tripolar mode. Their measurements, obtained with multiple electrodes in 16 ears, revealed that thresholds were lower for TP-C than for TP-A pulse trains in $22 \%$ $(48 / 219)$ of electrodes. This percentage was very similar to the corresponding value of $27 \%(9 / 33)$ in the present study. Another similarity is that they found a positive across-electrode correlation between the polarity effect and the threshold for a SYM-C pulse train, analogous to our correlation between the polarity effect and overall threshold. However, their correlation was quite weak, accounting only for about $5 \%$ of the variance. More recently, Goehring et al. (2018) used a method similar to that of Mesnildrey et al. (2017) to measure TP-A and TP-C thresholds in eight subjects. They also reported a weak positive correlation which, in their case, was not significant. Clearly, then, the size and significance of the correlation depend on the particular group of subjects and/ or electrodes studied. Indeed, inspection of Fig. 2 reveals that the correlation was largely driven by the results of subjects S22, C6, and (to a lesser extent) C3. In terms of our hypothesis, it may be that large correlations occur when the EMD is constant, and neural survival varies markedly, along the length of the cochlea. More generally, it is worth noting that although the across-electrode comparisons reported here and elsewhere involve a reasonably high number of degrees of freedom, they are obtained from only a modest number of subjects.

With the above caveats, of particular interest in the study by Mesnildrey et al. (2017) is their analysis of CT scans from nine ears, which allowed them to derive an estimate of the electrode-modiolar distance (EMD) for each subject. Although the EMD accounted for between 63 and $68 \%$ of the between-subject variance in thresholds, it accounted for only a small proportion of the between-electrode variance. The authors suggested that this was due to EMD varying substantially between but not within ears. They also showed that the EMD did not correlate significantly with the polarity effect. This is important for the interpretation of the present results because it is possible that the site of excitation, and hence the polarity effect, was affected by the EMD. If such a correlation had been found, this would have provided an alternative to our interpretation that the correlation between the polarity effect and overall thresholds was mediated by neural survival.

Zhou and Pfingst (2016) also obtained thresholds for multiple electrodes and estimated EMDs from the 
data relating EMD to electrode number reported by Long et al. (2014). They did not report the correlation directly but did state that MPI, defined as the difference between thresholds for 80- and 640-pps pulse trains, correlated with the EMD. Their data showed generally greater across-electrode threshold variations at 80 pps than at $640 \mathrm{pps}$, and so it is likely that the variation in the MPI was driven mainly by the 80-pps thresholds. If so, then those thresholds would have correlated positively with the EMD. However, they do not state how much of the variation in 80-pps thresholds was accounted for by EMD, nor whether a substantial across-electrode (rather than acrosslistener) correlation was observed. In addition, it is possible, as suggested by Mesnildrey (2017), that the across-electrode variation in EMD for a given subject may be smaller for Advanced Bionics listeners (as tested here) than for the Cochlear CI24RE listeners studied by Zhou and Pfingst (2016) and by Long et al. (2014). Mesnildrey reported that the average range of EMDs, across listeners, was $0.75 \mathrm{~mm}$ in his study compared to $1.2 \mathrm{~mm}$ in Long et al.'s study; an intermediate value of $1.00 \mathrm{~mm}$ is revealed by an analysis of the 10 Advanced Bionics listeners studied by DeVries et al. (2016).

\section{Relationship to Supra-Threshold Tasks}

Table 3 shows the across-subject correlations between the two measures obtained here-average thresholds and the polarity effect-and the supra-threshold measures reported previously by our laboratories (Bierer et al. 2015; Cosentino et al. 2016). Two correlations were statistically significant after Bonferroni correction: average thresholds vs. log GDTs, and the polarity effect vs RDR400 (which is a measure of the upper limit of temporal pitch). This does not mean, though, that log GDTs are more strongly related to average thresholds than to polarity, or that RDR400 is more strongly correlated with the polarity effect than with average thresholds. Such a conclusion would require that these two correlations were significantly larger than, respectively, those between polarity vs $\log$ GDT and average threshold vs. $\log 400$ R. This was not the case (Williams test, $\mathrm{df}=$ 5, two-tailed $p=0.07$ and 0.14 for GDT and RDR400 respectively). The safest conclusion is that both low average thresholds and a small or negative polarity effect correlate with good performance on some supra-threshold tasks, including gap detection and a measure (RDR400) of the upper limit of temporal pitch. There is also evidence from Mesnildrey et al. (2017) that, across subjects, small or negative polarity effects correspond to low thresholds on a spectrotemporal ripple test (Aronoff and Landsberger 2013). In addition, Zhou and Pfingst (2016) showed that high thresholds for 80-pps pulse trains-similar to the rate used here-correlated significantly, both across subjects and electrodes, with poor spatial selectivity as measured using forward masking. A caveat is that they measured spatial selectivity by measuring the slope relating masker position to the amount of masking of a fixed probe. This measures the spread of excitation produced by the maskers rather than by the signal.

Finally, it is worth noting that Zhou (2017) has recently observed substantial and significant improvements, both in speech perception and on a spectrotemporal ripple test, by deactivating electrodes with high low-rate thresholds. As they have pointed out, such high thresholds may reflect a combination of large EMD and poor local neural survival. If, as we have argued, the polarity effect is more affected by local neural survival (specifically that of the peripheral processes), then this may have two implications for the potential improvement of patient outcomes by reprogramming the clinical map. First, in devices such as the one studied here, where EMD variation across the array may be less than in the Cochlear CI24RE device, variations in spatial selectivity may be more strongly driven by neural survival, and it may be beneficial to have a measure that is specific to that parameter. Second, computational models (e.g., Goldwyn et al. 2010) indicate that, in devices that allow focused stimulation methods such as the tripolar mode, the appropriate intervention in cases of locally high thresholds depends on the cause. Specifically, Bierer and Litvak (2016) have argued that electrodes with a large EMD should be stimulated in tripolar mode, whereas those in neural "dead regions" should be deactivated. Again, a measure that differentiates between these two situations could provide significant clinical advantages.

\section{Summary and Conclusions}

(i) Consistent with recent reports, polarity sensitivity at threshold varied across listeners and electrodes. Specifically, thresholds were lower for TP-C than for TP-A pulses for $27 \%$ of the electrode-subject combinations tested here.

(ii) There was a significant trend for thresholds to be lower for more apical electrodes. However, the polarity effect did not vary significantly as a function of longitudinal electrode position.

(iii) There was a modest significant across-electrode correlation between the polarity effect and the average threshold for the two polarities. The direction of the correlation was that lower thresholds for TP-C than for TP-A pulses corresponded to lower average thresholds. The 
correlation was in the same direction but larger than observed in two recent reports.

(iv) Across subjects, the polarity effect correlated with the rate discrimination at high rates, as measured by Cosentino et al. (2016). The direction of the effect was that lower thresholds for TP-C than for TP-A pulses corresponded to better rate discrimination.

(v) The results are consistent with, but do not prove, the idea that the polarity effect is an indicator of good neural survival, which in turn influences detection thresholds and performance on some supra-threshold tasks.

\section{ACKNOWLEDGMENTS}

We thank Lindsay DeVries for generously providing the EMD measurements from her study.

Funding Information This research was supported by awards RG91365 (Medical Research Council, Carlyon), MC-A0605PQ75 (Action on Hearing Loss, Carlyon), and DC012142 (National Institute of Health, Arenberg).

\section{COMPLIANCE WITH ETHICAL STANDARDS}

All procedures were approved by the respective Human Subjects Review Boards.

Open Access This article is distributed under the terms of the Creative Commons Attribution 4.0 International License (http://creativecommons.org/licenses/by/4.0/), which permits unrestricted use, distribution, and reproduction in any medium, provided you give appropriate credit to the original author(s) and the source, provide a link to the Creative Commons license, and indicate if changes were made.

\section{REFERENCES}

Aronoff JM, Landsberger DM (2013) The development of a modified spectral ripple test. J Acoust Soc Am 134:EL217-EL222

BIERER J (2007) Threshold and channel interaction in cochlear implant users: evaluation of the tripolar electrode configuration. J Acoust Soc Am 121:1642-1653

Bierer JA, Faulkner KF (2010) Identifying cochlear implant channels with poor electrode-neuron interface: partial tripolar, single-channel thresholds and psychophysical tuning curves. Ear Hear 32:633-633

BieRER JA, Litvak L (2016) Reducing channel interaction through cochlear implant programming may improve speech perception: current focusing and channel deactivation. Trends Hear 20:233121651665338

Bierer JA, Deeks JM, Billig AJ, Carlyon RP (2015) Comparison of signal and gap-detection thresholds for focused and broad Cochlear implant electrode configurations. Jaro-J Assoc Res Otolaryngol 16:273-284
Bland JM, Altman DG (1995) Calculating correlation coefficients with repeated observations: part 1-correlation within subjects. $\mathrm{Br}$ Med J 310:446

Carlyon RP, Deeks JM (2015) Combined neural and behavioural measures of temporal pitch perception in cochlear implant users. J Acoust Soc Am 138:2885-2905

Carlyon RP, Deeks JM, Macherey O (2013) Polarity effects on place pitch and loudness for three cochlear-implant designs and at different cochlear sites. J Acoust Soc Am 134:503-509

Cosentino S, Deeks JM, Carlyon RP (2015) Procedural factors that affect measures of spatial selectivity in cochlear implant users. Trends Hear 19:1-16

Cosentino S, Carlyon RP, Deeks JM, Parkinson W, Bierer JA (2016) Rate discrimination, gap detection and ranking of temporal pitch in cochlear implant users. J Assoc Otolaryngol 17:371-382

DeVries L, Scheperle R, Bierer JA (2016) Assessing the electrodeneuron interface with the electrically evoked compound action potential, electrode position, and behavioral thresholds. J Assoc Res Otolaryngol 17:237-252

Fu Q-J (2002) Temporal processing and speech recognition in cochlear implant users. Neuroreport 13:1-5

Garadat SN, Zwolan TA, Pfingst BE (2012) Across-site patterns of modulation detection: relation to speech recognition. J Acoust Soc Am 131:4030-4041

Garadat SN, Zwolan TA, Pfingst BE (2013) Using temporal modulation sensitivity to select stimulation sites for processor MAPs in cochlear implant listeners. Audiol Neuro-Otol 18:247260

Goehring T, Arenberg JG, Deeks JM, Carlyon RP (2018) Effect of a channel-selection strategy based on polarity sensitivity on speech perception by cochlear implant users. Assoc Res Otolaryngol Abs 41:42

Goldwyn JH, Bierer SM, Bierer JA (2010) Modeling the electrodeneuron interface of cochlear implants: effects of neural survival, electrode placement, and the partial tripolar configuration. Hear Res 268:93-104

Hartmann R, Topp G, Klinke R (1984) Discharge patterns of cat primary auditory fibers with electrical-stimulation of the cochlea. Hear Res 13:47-62

Holden LK, Finley CG, Firszt JB, Holden TA, Brenner C, Potts LG, Gotter BD, Vanderhoof SS, Mispagel K, Heydebrand G, Skinner MW (2013) Factors affecting open-set word recognition in adults with cochlear implants. Ear Hear 34:342-360

Johnsson LG, Hawkins JE JR, Kingsley TC, Black FO, Matz GJ (1981) Aminoglycoside-induced cochlear pathology in man. Acta Otolaryngol Suppl 383:1-19

Long CJ, Holden TA, McClelland GH, Parkinson WS, Shelton C, Kelsall DC, Smith ZM (2014) Examining the electro-neural interface of cochlear implant users using psychophysics, CT scans, and speech understanding. J Assoc Res Otolaryngol 15:293-304

Macherey O, Wieringen A, Carlyon RP, Deeks JM, Wouters J (2006) Asymmetric pulses in cochlear implants: effects of pulse shape, polarity and rate. JARO 7:253-266

Macherey O, Carlyon RP, Deers JM, van Wieringen A, Wouters J (2008) Higher sensitivity of human auditory nerve fibers to positive electrical currents. Jaro-J Assoc Res Otolaryngol 9:241251

Macherey O, Deeks JM, Carlyon RP (2011) Extending the limits of place and temporal pitch perception in cochlear implant users. JARO 12:233-251

Macherey O, Carlyon RP, Chatron J, Roman S (2017) Effect of pulse polarity on thresholds and on non-monotonic loudness growth in cochlear implant users. J Assoc Res Otolaryngol 18:513-527. https://doi.org/10.1007/s10162-016-0614-4

Mesnildrey Q (2017) Towards a better understanding of the cochlear implant In: Aix-Marseielle 
Mesnildrey Q Macherey O, Carlyon RP, Venail F (2017) Polarity sensitivity in cochlear implants: relation with neural survival? In: Conference on implantable auditory prostheses, p 197. Lake Tahoe, CA, USA

Miller CA, Abbas PJ, Robinson BK, Rubinstein JT, Matsuoka AJ (1999) Electrically evoked single-fiber action potentials from cat: responses to monopolar, monophasic stimulation. Hear Res 130:197-218

Miller CA, Abbas PJ, Hay-McGutcheon MJ, Robinson BK, Nourski KV, Jeng FC (2004) Intracochlear and extracochlear ECAPs suggest antidromic action potentials. Hear Res 198:75-86

NAdol JB (1997) Patterns of neural degeneration in the human cochlea and auditory nerve: implications for cochlear implantation. Otolaryngol Head Neck Surg 117:220-228

OldHAm PD (1962) A note on the analysis of repeated measurements of the same subjects. J Chronic Diseases 15:969-977

PFingst BE, Xu L (2004) Across-site variation in detection thresholds and maximum comfortable loudness levels for cochlear implants. Jaro-Journal of the Association for Research in Otolaryngology 5:11-24

RANCK JB (1975) Which elements are excited in electrical stimulation of mammalian nervous system: a review. Brain Res 98:417440

RatTay F (1999) The basic mechanism for the electrical stimulation of the nervous system. Neuroscience 89:335-346

Rattay F, Lutter P, Felix H (2001) A model of the electrically excited human cochlear neuron I. Contribution of neural substructures to the generation and propagation of spikes. Hear Res 153:43-63
Tu Y-K, GiLthorpe MS (2007) Revisiting the relation between change and initial value: a review and evaluation. Statist Med 26:443-457

Undurraga JA, Wieringen A, Carlyon RP, Macherey O, Wouters J (2010) Polarity effects on neural responses of the electrically stimulated auditory nerve at different cochlear sites. Hear Res 269:146-161

van Wieringen A, Macherey O, Carlyon RP, Deeks JM, Wouters J (2008) Alternative pulse shapes in electrical hearing. Hear Res 242:154-163

WILSON BS (1997) The future of cochlear implants. Brit J of Audiol 31:205-225

Won JH, Clinard CG, Kwon S, Dasika VK, Nie K, Drennan WR, Tremblay KL, Rubinstein JT (2011) Relationship between behavioral and physiological spectral-ripple discrimination. Jaro-J Assoc Res Otolaryngol 12:375-393

Zноu N (2017) Deactivating stimulation sites based on low-rate thresholds improves spectral ripple and speech reception thresholds in cochlear implant users. J Acoust Soc Am 141:EL243-EL248

Zhou N, Pfingst BE (2014) Relationship between multipulse integration and speech recognition with cochlear implants. J Acoust Soc Am 136:1257-1268

Zhou N, Pfingst BE (2016) Evaluating multipulse integration as a neural-health correlate in human cochlear-implant users: relationship to spatial selectivity. J Acoust Soc Am 140:1537-1547

Zhou N, Kraft C, Colesa D, Pfingst B (2015) Integration of pulse trains in humans and guinea pigs with cochlear implants. Jaro-J Assoc Res Otolaryngol 16:523-534

Zimmermann CE, Burgess BJ, Nadol JB (1995) Patterns of degeneration in the human cochlear nerve. Hear Res 90:192-201 